\title{
Strangeness and charm at FAIR
}

\author{
Laura Tolos* \\ FIAS. Goethe-Universität Frankfurt am Main, \\ Ruth-Moufang-Str. 1, 60438 Frankfurt am Main, Germany \\ and \\ Theory Group, KVI, University of Groningen, \\ Zernikelaan 25, 9747 AA Groningen, The Netherlands \\ E-mail: tolos@kvi.nl
}

\section{Daniel Cabrera}

Departamento de Física Teórica II, Universidad Complutense,

28040 Madrid, Spain

\section{Daniel Gamermann, Raquel Molina and Eulogio Oset}

Departamento de Física Teórica and IFIC, Centro Mixto Universidad de Valencia-CSIC, Institutos de Investigación de Paterna, Aptdo. 22085, 46071 Valencia, Spain

\section{Tetsuro Mizutani}

Department of Physics, Virginia Polytechnic Institute and State University,

Blacksburg, VA 24061, USA

\section{Angels Ramos}

Departament d'Estructura i Constituents de la Matèria

Universitat de Barcelona, Diagonal 647, 08028 Barcelona, Spain

We study the properties of strange and charm mesons in hot and dense matter within a selfconsistent coupled-channel approach for the experimental conditions of density and temperature expected for the CBM experiment at FAIR/GSI. The in-medium solution at finite temperature accounts for Pauli blocking effects, mean-field binding on all the baryons involved, and meson self-energies. In the strange sector, the $\bar{K}$ spectral function spreads over a wide range of energies, reflecting the melting of the $\Lambda(1405)$ resonance and the contribution of $\left(\Lambda, \Sigma, \Sigma^{*}\right) N^{-1}$ components at finite temperature. In the case of charm mesons, the dynamically-generated $\Lambda_{c}(2593)$ and $\Sigma_{c}(2880)$ resonances remain close to their free-space position while acquiring a remarkable width. As a result, the $D$ meson spectral density shows a single pronounced peak for energies close to the $D$ meson free-space mass that broadens with increasing matter density with an extended tail particularly towards lower energies. We also discuss the implications for the $D_{s 0}(2317), D_{0}(2400)$ and the predicted $X(3700)$ resonances at FAIR energies.

8th Conference Quark Confinement and the Hadron Spectrum September 1-6 2008

Mainz, Germany

\footnotetext{
${ }^{*}$ Speaker.
} 


\section{Introduction}

Strangeness has been a matter of study over the last years in connection to exotic atoms [1] as well as heavy-ion collisions [2]. The analysis of the $\bar{K}$ interaction in nuclei has revealed some interesting features. Phenomenology of kaonic atoms shows that the $\bar{K}$ feels an attractive potential at low densities. This attraction results from the modified $s$-wave $\Lambda(1405)$ resonance in the medium due to Pauli blocking effects [3] together with the self-consistent consideration of the $\bar{K}$ self-energy [4] and the inclusion of self-energies of the mesons and baryons in the intermediate states [5]. Attraction of the order of $-50 \mathrm{MeV}$ at normal nuclear matter density $\rho_{0}=0.17 \mathrm{fm}^{-3}$ is obtained by different approaches, such as unitarizated chiral theories in coupled-channels [5]. Higher-partial waves have been studied $[6,7,8,9]$ and shown to be relevant for heavy-ion collisions. At present, relativistic heavy-ion experiments at beam energies below $2 \mathrm{AGeV}$ [2] have been testing strange mesons not only in a dense but also in a hot medium.

In this context, the charm degree of freedom has become a recent topic of analysis. The future CBM (Compressed Baryon Matter) experiment of the FAIR (Facility of Antiproton and Ion Research) project at GSI [10] will investigate, among others, the modification of the properties of open and hidden charm mesons in a hot dense baryonic environment. The in-medium modification of the $D(\bar{D})$ mesons may explain, for example, the $J / \Psi$ suppression, on the basis of a mass reduction of $D(\bar{D})$ in the nuclear medium. However, a self-consistent coupled-channel calculation is needed due to the strong coupling among the $D N$ and other meson-baryon channels $[11,12,13]$, which induces the appearance of dynamically-generated resonances.

In this paper we study the properties of strange $(\bar{K})$ and charm $(D)$ mesons in a hot nuclear medium pursuing a self-consistent coupled-channel procedure. Moreover, we analyze the effect of the self-energy of $D$ mesons on dynamically-generated charm and hidden charm scalar resonances.

\section{Strange and charm mesons in hot and dense matter}

The self-energy and, hence, the spectral function at finite temperature for strange $(\bar{K})$ and charm $(D)$ mesons is obtained using a self-consistent coupled-channel procedure.

For $\bar{K}$, a chiral unitary approach in coupled channels is performed, which incorporates the $s$ and $p$-waves of the kaon-nucleon interaction. The $s$-wave amplitude is obtained from the WeinbergTomozawa term of the $S U$ (3) chiral lagrangian. Unitarization in coupled channels is imposed by solving the Bethe-Salpeter equation with on-shell amplitudes. The model generates dynamically the $\Lambda(1405)$ resonance in the $I=0$ channel. The in-medium solution at finite temperature of the $s$-wave amplitude accounts for Pauli-blocking effects, mean-field binding on the nucleons and hyperons via a temperature-dependent $\sigma-\omega$ model, and the dressing of the pion and antikaon through their corresponding self-energies. This requires a self-consistent evaluation of the $\bar{K}$ self-energies. The $p$-wave self-energy is obtained via the hyperon-hole $\left(Y N^{-1}\right)$ excitations (see Ref. [9]).

In the case of the $D$ meson, the multichannel Bethe-Salpeter equation is solved taking, as bare interaction, a type of broken SU(4) $s$-wave Tomozawa-Weinberg interaction supplemented by an attractive isoscalar-scalar term and using a cutoff regularization scheme. This cutoff is fixed by reproducing the position and the width of the $I=0 \Lambda_{c}(2593)$ resonance. As a result, a new resonance in $I=1$ channel $\Sigma_{c}(2880)$ is generated [12]. The in-medium solution at finite temperature 

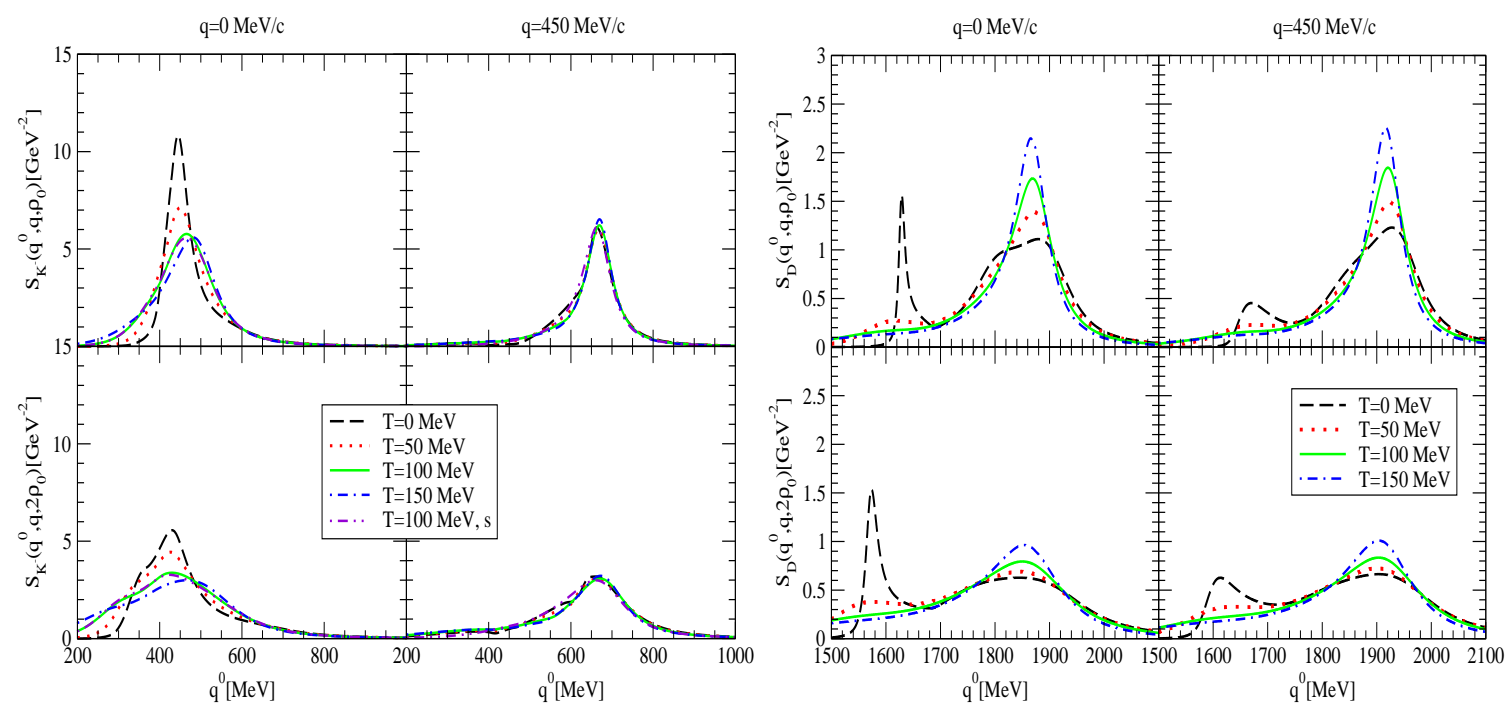

Figure 1: Spectral function for $\bar{K}$ (left) and $D$ (right) mesons.

incorporates, as well, Pauli blocking effects, baryon mean-field bindings and $\pi$ and $D$ meson selfenergies in a self-consistent manner (see Ref. [13]). The $p$-wave self-energy is also obtained via the corresponding $Y_{c} N^{-1}$ excitations [14].

For both cases, the self-energy is obtained self-consistently summing the transition amplitude $T$ for the different isospins over the nucleon Fermi distribution at given temperature, $n(\vec{q}, T)$, as

$$
\Pi\left(q_{0}, \vec{q}, T\right)=\int \frac{d^{3} p}{(2 \pi)^{3}} n(\vec{p}, T)\left[T^{(I=0)}\left(P_{0}, \vec{P}, T\right)+3 T^{(I=1)}\left(P_{0}, \vec{P}, T\right)\right],
$$

where $P_{0}=q_{0}+E_{N}(\vec{p}, T)$ and $\vec{P}=\vec{q}+\vec{p}$ are the total energy and momentum of the meson-nucleon pair in the nuclear matter rest frame, and $\left(q_{0}, \vec{q}\right)$ and $\left(E_{N}, \vec{p}\right)$ stand for the energy and momentum of the meson and nucleon, respectively, also in this frame. The spectral function then reads

$$
S\left(q_{0}, \vec{q}, T\right)=-\frac{1}{\pi} \frac{\operatorname{Im} \Pi\left(q_{0}, \vec{q}, T\right)}{\left|q_{0}^{2}-\vec{q}^{2}-m^{2}-\Pi\left(q_{0}, \vec{q}, T\right)\right|^{2}} .
$$

On the 1.h.s. of Fig. 1, the $\bar{K}$ spectral function is depicted. It shows a strong mixing between the quasi-particle peak and the $\Lambda(1405) N^{-1}$ and $Y N^{-1}$ excitations. The effect of the $p$-wave $Y N^{-1}$ subthreshold excitations is repulsive for the $\bar{K}$ potential, compensating in part the attraction from the $s$-wave $\bar{K} N$ interaction. Temperature softens the $p$-wave contributions to the spectral function at the quasi-particle energy. Moreover, together with the $s$-wave mechanisms, the $p$-wave self-energy provides a low-energy tail which spreads the spectral function considerably, due to the smearing of the nucleonic Fermi surface. Density dilutes the spectral function further.

The evolution with temperature and density of the $D$ meson spectral function is shown on the r.h.s of Fig. 1. We observe the dilution of the $\Lambda_{c}(2593) N^{-1}$ and $\Sigma_{c}(2880) N^{-1}$ structures with increasing temperature, while the quasiparticle peak gets closer to its free value becoming narrower. Therefore, the distribution of the spectral function concentrates around the quasiparticle energy, 

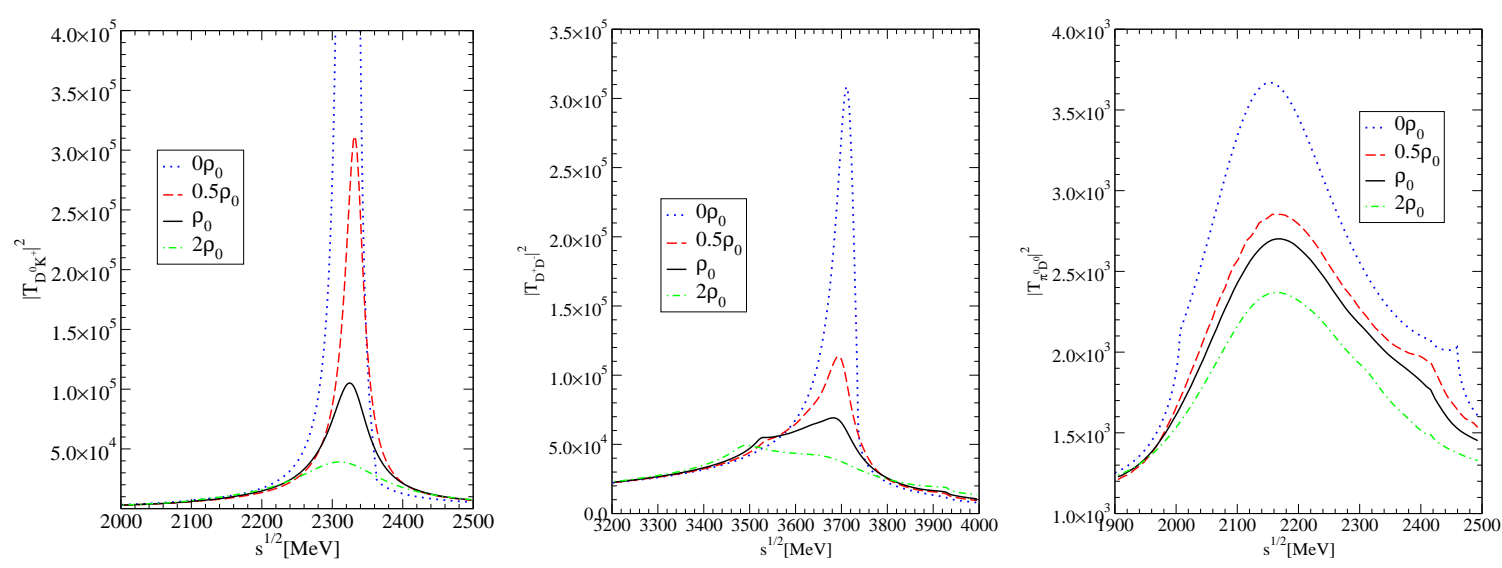

Figure 2: $D_{s 0}(2317)$ (left), $X(3700)$ (middle) and $D_{0}(2400)$ (right) resonances in dense matter.

although maintaining the overall strength in its lower energy part. As density increases, the quasiparticle peak broadens and the low-energy strength, associated to the $\Lambda_{c}(2593) N^{-1}$ components and related to $Y_{c} \pi N^{-1}, Y_{c} N N^{-2}, \ldots$ absorption modes, increases.

\section{Charm and hidden charm resonances in nuclear matter}

The renormalization of the properties of mesons in nuclear matter as, for example, the $D$ meson, can have important consequences on the medium modification of scalar mesons which are dynamically generated in the charm sector. Concretely, we shall study the medium modification of the $D_{s 0}(2317)$ and $D_{0}(2400)$. In addition, we also study the medium changes of a hidden charm scalar meson predicted in [15], the $X(3700)$, which might have been observed by Belle [16].

Those resonances are generated dynamically by solving the coupled-channel Bethe-Salpeter equation for two pseudoscalars. The kernel is derivated from an extrapolation to $S U(4)$ of the $S U(3)$ chiral Lagrangian used to generate the scalar mesons $\sigma(600), f_{0}(980), a_{0}(980)$ and $\kappa(900)$ in the light sector, but with the $S U(4)$ symmetry strongly broken, mostly due to the explicit consideration of the masses of the vector mesons exchanged between pseudoscalars [17].

The $D_{s 0}(2317)$ mainly couples to $D K$ system, while the $D_{0}(2400)$ to $D \pi$. On the other hand, the hidden charm state $X(3700)$ couples most strongly to $D \bar{D}$. Therefore, any change in the $D$ meson properties in nuclear matter will have an important effect on those resonances. In Fig. 2, we obtain that in the case of the $D_{s 0}(2317)$ and $X(3700)$ resonances, which have a zero and small width, respectively, the medium effects lead to widths of the order of 100 or $200 \mathrm{MeV}$ at normal nuclear matter density, respectively. As for the $D_{0}(2400)$, we observe an extra widening from the already large width of the resonance in free space. However, the large original width makes the medium effects comparatively much weaker than for the other two resonances [14].

The study of the width of those resonances and the medium reactions contributing to it provides information on the features of the resonances and the self-energy of the $D$ meson in a nuclear medium. We suggest to look at transparency ratios to investigate those in-medium widths.

In conclusion, the experimental analysis of those properties is a valuable test of the dynamics of the $D$ meson interaction with nucleons and nuclei, and the nature of the charm and hidden charm 
scalar resonances, all of them topics which are subject of much debate at present. The results obtained here should stimulate experimental work in hadron facilities, in particular at FAIR [10], where the investigation of charm physics is one of the priorities.

\section{References}

[1] E. Friedman and A. Gal, In-medium nuclear interactions of low-energy hadrons, Phys. Rept. 452 (2007) 89 [arXiv:0705.3965[nucl-th]].

[2] C. Fuchs, Kaon production in heavy ion reactions at intermediate energies, Prog. Part. Nucl. Phys. 56 (2006) 1 [arXiv:nucl-th/0507017].

[3] V. Koch, $K^{-}$- proton scattering and the Lambda (1405) in dense matter, Phys. Lett. B 337 (1994) 7 [arXiv:nucl-th/9406030].

[4] M. Lutz, Nuclear kaon dynamics, Phys. Lett. B 426 (1998) 12 [arXiv:nucl-th/9709073].

[5] A. Ramos and E. Oset, The properties of anti-K in the nuclear medium, Nucl. Phys. A 671 (2000) 481 [arXiv:nucl-th/9906016].

[6] L. Tolos, A. Ramos, A. Polls and T. T. S. Kuo, Partial wave contributions to the antikaon potential at finite momentum, Nucl. Phys. A 690 (2001) 547 [arXiv:nucl-th/0007042]; L. Tolos, A. Ramos and A. Polls, The antikaon nuclear potential in hot and dense matter, Phys. Rev. C 65 (2002) 054907 [arXiv:nucl-th/0202057].

[7] L. Tolos, A. Ramos and E. Oset, Chiral approach to antikaon s- and p-wave interactions in dense nuclear matter, Phys. Rev. C 74 (2006) 015203 [arXiv:nucl-th/0603033].

[8] M. F. M. Lutz, C. L. Korpa and M. Moller, Antikaons and hyperons in nuclear matter with saturation, Nucl. Phys. A 808 (2008) 124 [arXiv:0707.1283 [nucl-th]].

[9] L. Tolos, D. Cabrera and A. Ramos, Strange mesons in nuclear matter at finite temperature, Phys. Rev. C 78 (2008) 045205 [arXiv:0807.2947 [nucl-th]].

[10] http://www.gsi.de/fair/index.html

[11] L. Tolos, J. Schaffner-Bielich and A. Mishra, Properties of D-mesons in nuclear matter within a self-consistent coupled-channel approach, Phys. Rev. C 70 (2004) 025203 [arXiv:nucl-th/0404064]; L. Tolos, J. Schaffner-Bielich and H. Stoecker, D-mesons: In-medium effects at FAIR, Phys. Lett. B 635 (2006) 85 [arXiv:nucl-th/0509054].

[12] M. F. M. Lutz and C. L. Korpa, Open-charm systems in cold nuclear matter, Phys. Lett. B 633 (2006) 43 [arXiv:nucl-th/0510006]; T. Mizutani and A. Ramos, D mesons in nuclear matter: A DN coupled-channel equations approach, Phys. Rev. C 74 (2006) 065201 [arXiv:hep-ph/0607257].

[13] L. Tolos, A. Ramos and T. Mizutani, Open charm in nuclear matter at finite temperature, Phys. Rev. C 77 (2008) 015207 [arXiv:0710.2684 [nucl-th]].

[14] R. Molina, D. Gamermann, E. Oset and L. Tolos, Charm and hidden charm scalar mesons in the nuclear medium, arXiv:0806.3711 [nucl-th].

[15] D. Gamermann and E. Oset, Hidden charm dynamically generated resonances and the $e^{+} e^{-} \rightarrow J / \psi D \bar{D}, J / \psi D \bar{D}^{*}$ reactions, Eur. Phys. J. A 36 (2008) 189 [arXiv:0712.1758 [hep-ph]].

[16] K. Abe et al. [Belle Collaboration], Search for new charmonium states in the processes $e^{+} e^{-} \rightarrow J / \psi D^{(*)} D^{(*)}$ at $\sqrt{s} \sim 10.6 \mathrm{GeV}$, arXiv:0708.3812 [hep-ex].

[17] D. Gamermann, E. Oset, D. Strottman and M. J. Vicente Vacas, Dynamically Generated Open and Hidden Charm Meson Systems, Phys. Rev. D 76 (2007) 074016 [arXiv:hep-ph/0612179]. 